\title{
In Vivo Identification of a Negative Regulatory Element in the Mouse Renin Gene Using Direct Gene Transfer
}

Takehiko Yamada, Masatsugu Horiuchi, Ryuichi Morishita, Lunan Zhang, Richard E. Pratt, and Victor J. Dzau

Falk Cardiovascular Research Center, Stanford University School of Medicine, Stanford, California 94305-5246

\begin{abstract}
DBA/2J mouse contains two renin gene loci $\left(\operatorname{Ren} 1^{d}\right.$ and $\left.\operatorname{Ren} 2^{d}\right) . \operatorname{Ren} 2^{d}$ but not $\operatorname{Ren} 1^{d}$ is expressed in submandibular gland (SMG) while both are expressed in the kidney. Based on in vitro studies, we have postulated that a negative regulatory element (NRE) in the renin gene promoter is involved in its tissue-specific expression. In this study, we examined the molecular mechanism at the in vivo level using direct gene transfer. Fragments of the Ren1 ${ }^{d}$ or Ren2 ${ }^{d}$ promoter were fused to a chloramphenicol acetyltransferase (CAT) gene expression vector. These constructs complexed in fusogenic liposomes were injected directly into the mouse SMG or intraarterially into the mouse kidney via the renal artery. The vector containing the CAT exhibited readily detectable in vivo expressions in both SMG and kidney. In the SMG, $\operatorname{Ren}^{d}{ }^{d}$ fragment containing the NRE abolished CAT expression while deletion of the NRE restored CAT expression. The homologous fragment from the Ren $2^{d}$ promoter did not inhibit CAT expression while deletion of the 150-bp insertion resulted in the inhibition. Cotransfection of Ren $1^{d}$ construct with $\operatorname{Ren} 1^{d}$-NRE oligonucleotides as transcriptional factor decoy restored CAT expression. Contrary to the SMG, transfection with $\operatorname{Ren}^{d}{ }^{d}$ fragment-CAT construct or $\operatorname{Ren}^{d}{ }^{d}$ fragment-CAT construct into the kidney resulted in similar levels of CAT expression. Interestingly, human cmyc NRE oligonucleotides which share homology with Ren $1^{d}$-NRE competed effectively with these oligonucleotides for the regulation of $\operatorname{Ren} 1^{d}$ gene expression in vivo. This NRE sequence is also homologous to silencer elements found in multiple mammalian genes, suggesting the presence of a family of NRE/NRE binding proteins regulating expression of diverse genes. (J. Clin. Invest. 1995. 96:1230-1237.) Key words: submandibular gland $\cdot$ negative regulatory element - renin gene promoter • in vivo gene transfer • decoy
\end{abstract}

\section{Introduction}

Tissue-specific gene expression is dependent on the specific interaction of cis-DNA sequences with nuclear trans-activating proteins. Certain trans-acting factors are ubiquitous, whereas others exhibit temporal, tissue, and/or cell-specific expression.

Address correspondence to Victor J. Dzau, M.D., Falk Cardiovascular Research Center, Stanford University School of Medicine, 300 Pasteur Drive, Stanford, CA 94305-5246. Phone: 415-723-5013; FAX: 415725-2178.

Received for publication 4 October 1994 and accepted in revised form 18 May 1995.

J. Clin. Invest.

(c) The American Society for Clinical Investigation, Inc. 0021-9738/95/09/1230/08 $\$ 2.00$

Volume 96, September 1995, 1230-1237
Recently, it has been shown that the interaction of a negative regulatory element (NRE or silencer) ${ }^{1}$ with specific nuclear trans-acting proteins plays an important role in tissue-specific regulation of gene expression such as IL-2 gene or T cell receptor gene $(1,2)$. Further complexity is attained when multiple trans-acting factors, especially those exerting opposing effects, are able to bind to the same cis-element. In this case, competition for the same element can play an important role in gene regulation.

Renin, an aspartyl proteinase involved in the regulation of cardiovascular homeostasis, exhibits tissue-specific expression $(3,4)$. We have focused our studies on the molecular mechanism of tissue-specific renin gene expression in mice. Certain strains of mice such as DBA/2J contain two renin gene loci $\left(\operatorname{Ren} 1^{d}\right.$ and $\left.\operatorname{Ren} 2^{d}\right)$, which show marked tissue specific expression (5). For example, Ren $1^{d}$, the homologue of the human and rat renin gene, is expressed in the kidney but not in the submandibular gland (SMG). On the other hand, Ren2 ${ }^{d}$ is expressed in both the kidney and SMG. Our previous in vitro studies using promoter-reporter gene chimeras as well as gel mobility shift assays suggest that tissue-regulated expression is dependent in part on the specific interaction of an NRE located in the 5 '-flanking region of the renin gene with NRE binding proteins (NREB) $(6,7)$ (Fig. 1). Our in vitro data suggested that in the kidney the effect of the NREB is attenuated by the competitive binding of cAMP responsive element (CRE) binding proteins (CREB). In extrarenal tissues such as the SMG, CREB appears to be inactivated (7). Consequently, the NREB is able to bind to the NRE, resulting in the silencing of Ren $1^{d}$ in the SMG. On the other hand, Ren $2^{d}$ is expressed in the SMG because the NRE appears to be nonfunctional due to a 150-bp insertion that interrupts the NRE (8).

These data were derived from biochemical or cell culture experiments using noncognate cells that require validation in vivo. An ideal approach is to use promoter-reporter gene chimeric plasmids in transgenic mice. However, this method is time consuming and species limited. To circumvent these problems, in this study we use a novel approach using in vivo gene transfer of promoter-reporter chimeras in combination with transcriptional factor decoy oligonucleotides. The transcriptional factor decoy method is based on the principle of competition for binding proteins between the cis-elements present in a target gene and exogenously added double-stranded decoy oligonucleotides corresponding to that $c i s$-sequence. Several investigators have used this approach to block the activity of multiple transcriptional factors $(9,10)$.

We have recently used a highly efficient and simple method

1. Abbreviations used in this paper: CAT, chloramphenicol acetyltransferase; CRE, cAMP responsive element; CREB, CRE binding protein; HMG 1, high mobility group 1 protein; HVJ, hemagglutinating virus of Japan; MHC-I, major histocompatibility complex class-I; NRE, negative regulatory element; NREB, NRE binding protein; SMG, submandibular gland; $\mathrm{tk}$, thymidine kinase. 


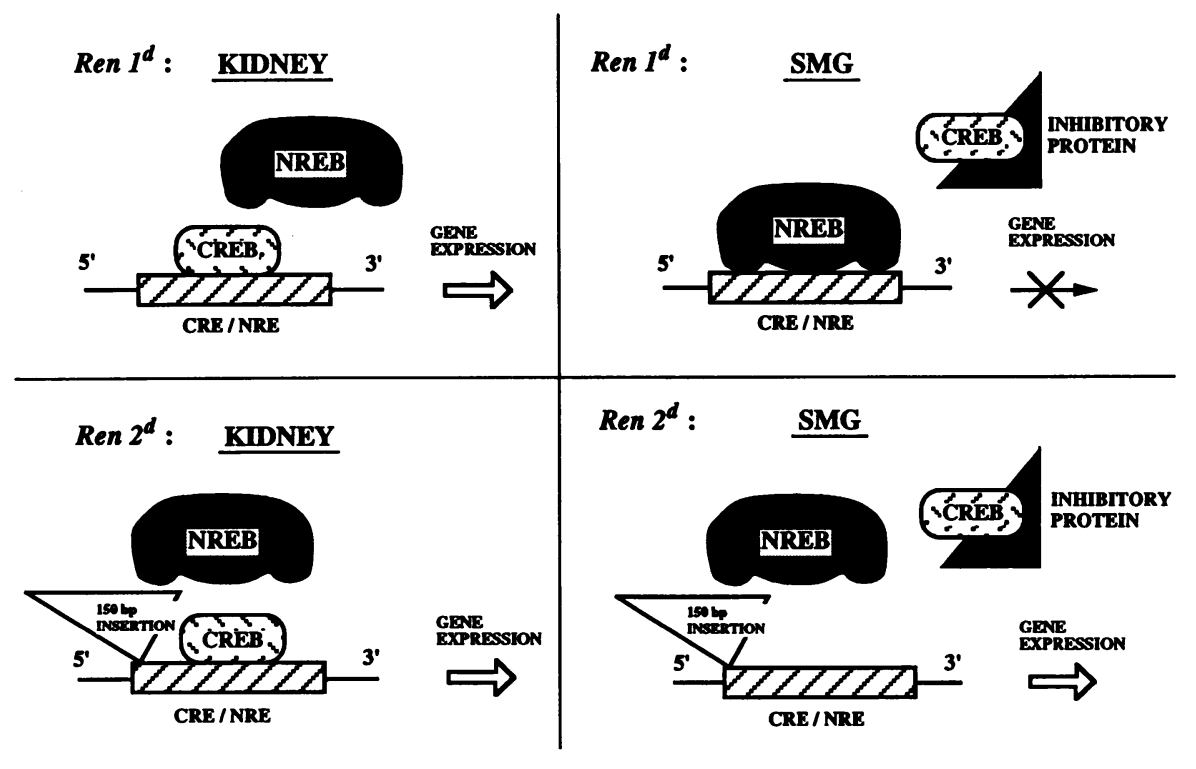

Figure 1. Schematic diagram depicting our hypothesis of the molecular mechanisms influencing mouse renin gene expression in the SMG and kidney. The hatched bars represent the CRE/NRE region present in the mouse renin gene. The CREB and the NREB compete for binding to this region with the CREB having a greater affinity. Ren $I^{d}$ gene expression is suppressed in the SMG because CREB is sequestered by an inhibitory protein, thereby permitting NREB to bind to NRE sequence. The triangles represent the 150-bp insert present in the Ren $2^{d}$ gene, which inhibits NREB binding to the NRE, allowing Ren $2^{d}$ expression in both tissues. of DNA transfer using the hemagglutinating virus of Japan (HVJ), also known as Sendai virus. This method involves the encapsulation of DNA in neutral liposomes which is then complexed with ultraviolet-inactivated HVJ (11). The HVJ enhances the fusion of the liposomes with cell membranes, facilitating the intracellular delivery of the DNA. A further modification, complexing the DNA with high mobility group 1 protein (HMG 1), increases the transport of the DNA into the nucleus. This technique possesses many ideal properties for gene transfer such as efficiency, safety, simplicity, brevity of incubation time, no limitations of inserted DNA size, and efficiency in multiple animal species including rodent and rabbit. The HVJ method has been successfully used for gene transfer in vivo into various tissues including liver (11), kidney (12), and vascular wall (13) as well as cells in culture (14). This method has allowed us to transfer plasmid vector DNA and oligonucleotides efficiently. To demonstrate that the NRE found in the renin gene promoter region is involved in regulation of gene expression in vivo, we transfected renin gene promoter-chloramphenicol acetyltransferase (CAT) fusion constructs directly into the SMG and kidney in vivo. In the SMG study, we further transfected the constructs with or without NRE oligonucleotides as transcriptional factor decoy to inhibit the interactions between the renin-promoter constructs (NRE) and endogenous transcriptional factors (NREB) in vivo.

\section{Methods}

Plasmid constructions (Fig. 2). pUtkCAT is a mammalian expression vector encoding CAT under the control of a truncated herpes simplex virus thymidine kinase (tk) promoter (15). p0CAT is a vector containing a CAT gene but no eukaryotic promoter (15). pUtkCAT-Ren1 includes a 340-bp XbaI fragment from Ren ${ }^{d}$ gene (positions -707 to -368) which contains the NRE, cloned upstream of the tk promoter. pUtkCAT-Ren 2 includes a corresponding 484-bp XbaI fragment from the Ren $2^{d}$ gene (positions -1058 to -575 ). pUtkCAT-Ren1 $\triangle$ NRE is a deletion mutant plasmid that lacks the 23-bp segment (positions -619 to -597) of the NRE sequence from pUtkCAT-Ren 1 constructed by PCR using the overlap-extension method which uses PCR primers to loop out the desired sequences (6). pUtkCAT-Ren $2 \Delta 150$ is a deletion mutant plasmid that lacks the 150-bp insertion (positions -969 to -820 ) form pUtkCAT-Ren 2 constructed by PCR. To monitor the efficiency of transfection, pGL2-CV, a control vector that contains an SV40 promoter and enhancer fused to a luciferase gene (Promega, Madison, WI) was cotransfected with the above renin-CAT constructs. All plasmids for in vivo transfection used in this study were purified on cesium chloride gradients.

Preparation of HVJ-liposomes. Mixture of lipids, plasmid DNA, nuclear protein, and virus was prepared as described previously (11). Phosphatidylserine, phosphatidylcholine, and cholesterol were dissolved in tetrahydrofuran in a weight ratio of 1:4.8:2. The lipid mixture (10 mg) was deposited on the sides of a flask by removal of the tetrahydrofuran in a rotary evaporator. To increase the transport of the DNA to the nucleus, HMG 1, purified from calf thymus, was complexed with the plasmid DNA mixture (renin-promoter CAT construct and pGL2-CV) by incubation at $4^{\circ} \mathrm{C}$ for $1 \mathrm{~h}$. The DNA-HMG 1 complex was then added to the dried lipid in $200 \mu \mathrm{l}$ of balanced salt (BSS; $137 \mathrm{mM} \mathrm{NaCl}, 5.4 \mathrm{mM}$ $\mathrm{KCl}, 10 \mathrm{mM}$ Tris- $\mathrm{HCl}$ [ $\mathrm{pH} 7.6]$ ). In the decoy study, double-stranded phosphorothioate oligonucleotides were also added in this mixture (15 $\mu \mathrm{M}$ final concentration). The liposome-DNA-HMG 1 complex suspension was prepared by vortexing followed by sonication for $3 \mathrm{~s}$ and shaking for $30 \mathrm{~min}$. Purified HVJ (Z strain, 10,000 hemagglutinating units ), inactivated by ultraviolet irradiation $\left(110 \mathrm{erg} / \mathrm{mm}^{2} / \mathrm{s}\right)$ for $3 \mathrm{~min}$ immediately before use, was added to the liposome suspension and incubated at $4^{\circ} \mathrm{C}$ for $10 \mathrm{~min}$ and then for 30 min with gentle shaking at $37^{\circ} \mathrm{C}$. Free $\mathrm{HVJ}$ was removed from the $\mathrm{HVJ}$-liposomes by sucrose density gradient centrifugation. The top layer of the sucrose gradient containing the HVJ-liposomes complex was collected and mixed with $2 \mathrm{mM} \mathrm{CaCl}{ }_{2}$ and $1 \mathrm{mg} / \mathrm{ml}$ glucose.

In vivo transfection and tissue harvest. Male $\mathrm{DBA} / 2 \mathrm{~J}$ mice (40d-old) (Charles River Breeding Laboratories, Cambridge, MA) were anesthetized using ketamine $(80 \mathrm{mg} / \mathrm{kg}$ ) (Parke-Davis, Morris Plains, $\mathrm{NJ})$ and xylazine $(12 \mathrm{mg} / \mathrm{kg}$ ) (Lloyd Laboratories, Shenandoah, IA) and underwent either SMG or kidney transfection procedure. In the SMG transfection study, $100 \mu \mathrm{l}$ of the HVJ-liposomes complex (prepared as above) was injected directly into 10 different sites of SMG (an organ of $\sim 160 \mu \mathrm{g}$ ) using a 31-gauge needle. In the kidney transfection study, abdominal aorta was temporally clipped above the left renal artery and a needle was inserted directly into the left renal artery. The left kidney was flushed with saline, then $300 \mu \mathrm{l}$ of the HVJ-liposomes complex was injected slowly ( $5 \mathrm{~min}$ ) into the left kidney. The needle was then removed and the clip was released. The incision was closed and the animals were allowed to recover. $3 \mathrm{~d}$ after the transfection, mice were killed and the tissue ( $\mathrm{s}$ ) removed rapidly for further analysis. At the time of tissue harvest, there was no evidence of bleeding or inflammation.

CAT assays. CAT assays were performed as described previously by Seed et al. (16). In brief, fresh SMG or kidney was homogenized 

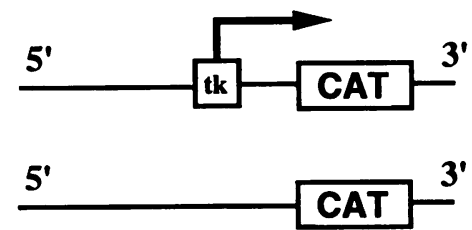

\section{: pUtkCAT}

: POCAT

Figure 2. Renin gene promoter-CAT expression plasmids. pUtkCAT (5.5k-bp) is a CAT expression vector containing a truncated herpes simplex virus thymidine kinase promoter. p0CAT (5.2k-bp) contains a CAT gene but no eukaryotic promoter. pUtkCAT-Ren1, derived from pUtkCAT, contains a 340-bp XbaI fragment from Renl ${ }^{d}$ gene including the putative CRE and NRE fused upstream to the thymidine kinase $(t k)$ promoter. pUtkCATRen2, also derived from pUtkCAT, contains a 484-bp XbaI fragment from the corresponding region in the Ren $2^{d}$ gene. pUtkCAT-Ren $1 \triangle N R E$ is a deletion mutant that lacks the 23-bp segment of NRE sequence from pUtkCAT-Ren1. pUtkCAT-Ren $2 \Delta 150$ is a deletion mutant plasmid that lacks the 150-bp insertion from pUtkCAT-Ren2.

with a Polytron (Brinkmann Instruments, Inc., Westbury, NY) in 300 or $800 \mu \mathrm{l}$ of $250 \mathrm{mM}$ Tris- $\mathrm{HCl}$ [pH 7.5], $0.5 \%$ Triton X-100, and 5 mM EDTA, respectively. After centrifugation at $12,000 \mathrm{~g}$ for $10 \mathrm{~min}$, $20 \mu \mathrm{l}$ of the supernatant was used in the luciferase assay. The remaining amount of the supernatant was incubated at $70^{\circ} \mathrm{C}$ for $15 \mathrm{~min}$ to inactivate endogenous acetylases. After a second centrifugation, the protein concentration was measured by the Bradford method (17) (Bio Rad Laboratories, Hercules, CA). The final supernatant was assayed for CAT activity. $200 \mu \mathrm{g}$ of protein per sample $(50-100 \mu \mathrm{l}), 5 \mu \mathrm{l}$ of $\left[{ }^{14} \mathrm{C}\right]$ chloramphenicol (specific activity, $56 \mathrm{mCi} / \mathrm{mmol}$; Amersham Corp., Arlington Heights, IL), and $8 \mu \mathrm{l}$ of $n$-butyryl coenzyme A $(5 \mathrm{mg} / \mathrm{ml}$; Sigma Chemical Co., St. Louis, MO) were mixed and filled to a total volume of $125 \mu \mathrm{l}$ with homogenization buffer. The reaction mixture was incubated at $37^{\circ} \mathrm{C}$ for $16 \mathrm{~h}$, which was in the linear range of the reaction. The acetylated chloramphenicol fraction of the suspension was extracted by adding $360 \mu \mathrm{l}$ xylene. Suspensions were back-extracted three times with $250 \mathrm{mM}$ Tris- $\mathrm{HCl}$ [pH 8.0]. Aliquots of $200 \mu \mathrm{l}$ were counted in scintillation fluid in a beta counter. Relative CAT activity (counts per minute/picogram of luciferase) was calculated by dividing the CAT activity in each gland (counts per minute/milligram of protein) by the corresponding levels of luciferase (picograms).

Luciferase assays. Luciferase assays were performed as described previously (18) using a luciferase assay system (Promega). In brief, light emission was measured for $20 \mathrm{~s}$ by Monolight luminometer (model 2001; Analytical Luminescence Laboratory, San Diego, CA) immediately after the addition of $100 \mu \mathrm{l}$ of luciferase assay reagent containing coenzyme A, D-luciferin, and ATP to $20 \mu \mathrm{l}$ of the supernatant of sample or standard luciferase (Sigma Chemical Co.).

Preparation of oligonucleotides. Oligonucleotides (28 bp) corresponding to the mouse Ren $1^{d}$-NRE (-617/-590), human c-myc NRE $(-336 /-309)(19)$, mouse major histocompatibility complex class-I (MHC-I) NRE (-165/-138) (20) were synthesized on an automated solid-phase synthesizer (Applied Biosystems, Inc., Foster City, CA): Ren $1^{d}$-NRE: $5^{\prime}$ CTAACTTGGTCTCACAGGCTAGAATTTA 3'; cmyc NRE: 5' ATGATTTATACTCACAGGACAAGGATGC 3'; MHCI NRE: 5' CCCCATCTCCTCAGTTTCACTTCTGCAC 3'; putative consensus NRE sequence (6) is underlined.

Single-stranded oligonucleotides were washed by $70 \%$ ethanol, dried, and dissolved in sterile Tris/EDTA buffer (10 mM Tris- $\mathrm{HCl}$ [pH
8.0], 1 mM EDTA). The oligonucleotides were purified over a NAP column (Pharmacia LKB, Piscataway, NJ) and quantitated by spectrophotometer. Complementary pairs of single-stranded oligonucleotide were annealed for $2 \mathrm{~h}$ while the temperature descended from 80 to $25^{\circ} \mathrm{C}$. We used double-stranded phosphorothioate oligonucleotides as a decoy to decrease the degradation of the oligomer during the experiment.

Preparation of nuclear extract. Nuclear extract was prepared from SMG as described previously (7). In brief, the SMG from 10 male DBA/2J mice (40-d-old, Charles River Breeding Laboratories) were removed quickly and homogenized with a Potter-Elvehjem homogenizer in $4 \mathrm{vol}$ of ice-cold homogenization buffer ( $10 \mathrm{mM}$ Hepes [pH 7.5], $0.5 \mathrm{M}$ sucrose, $0.5 \mathrm{mM}$ spermidine, $0.15 \mathrm{mM}$ spermin, $5 \mathrm{mM}$ EDTA, $0.25 \mathrm{mM}$ EGTA, $7 \mathrm{mM} \beta$-mercaptoethanol, $1 \mathrm{mM}$ PMSF). After centrifugation at $12,000 \mathrm{~g}$ for $30 \mathrm{~min}$ at $4^{\circ} \mathrm{C}$, the pellet was lysed in $1 \mathrm{vol}$ of ice-cold homogenization buffer containing $0.1 \%$ Nonidet $\mathrm{P}-40$ by homogenizing in a Dounce homogenizer. Then lysed nuclei were centrifuged at $12,000 \mathrm{~g}$ for $30 \mathrm{~min}$ at $4^{\circ} \mathrm{C}$ and the pellet nuclei were washed twice with ice-cold buffer containing $0.35 \mathrm{M}$ sucrose. After washing, nuclei were preextracted with $1 \mathrm{vol}$ of ice-cold homogenization buffer containing $0.05 \mathrm{M} \mathrm{NaCl}$ and $10 \%$ glycerol for $15 \mathrm{~min}$ at $4^{\circ} \mathrm{C}$. The nuclei were then extracted with homogenization buffer containing $0.3 \mathrm{M} \mathrm{NaCl}$ and $10 \%$ glycerol for $1 \mathrm{~h}$ at $4^{\circ} \mathrm{C}$ and the concentration of DNA was adjusted to $1 \mathrm{mg} / \mathrm{ml}$. After pelleting the extracted nuclei at $12,000 \mathrm{~g}$ for $30 \mathrm{~min}$ at $4^{\circ} \mathrm{C}$, the supernatant fraction was brought to $45 \%$ $\left(\mathrm{NH}_{4}\right)_{2} \mathrm{SO}_{4}$ and stirred for $30 \mathrm{~min}$ at $4^{\circ} \mathrm{C}$. The precipitated proteins were collected at $17,000 \mathrm{~g}$ for $30 \mathrm{~min}$, resuspended in homogenization buffer containing $0.35 \mathrm{M}$ sucrose, and stored in aliquots at $-70^{\circ} \mathrm{C}$.

Gel mobility shift assay. Double-stranded Ren $1^{d}$-NRE oligonucleotides and c-myc NRE oligonucleotides, which have cohesive end, were labeled as primers at the 3' end with the Klenow fragment of Escherichia coli DNA polymerase I (GIBCO BRL, Gaithersburg, MD) using $\left[\alpha{ }^{32} \mathrm{P}\right] \mathrm{dCTP}$ (sp act $=3,000 \mathrm{Ci} / \mathrm{mmol}$; Amersham Corp.). After end labeling, ${ }^{32} \mathrm{P}$-labeled oligonucleotides were purified by Nick column (Pharmacia LKB). Binding reactions $(10 \mu \mathrm{l})$ including each ${ }^{32} \mathrm{P}$-labeled primer $(0.5-1 \mathrm{ng}, 20,000 \mathrm{cpm})$ and $1 \mu \mathrm{g}$ of polydeoxyinosinic-polydeoxycytidic acid (Sigma Chemical Co.) were incubated with $10 \mu \mathrm{g}$ of SMG nuclear extracts for $30 \mathrm{~min}$ at room temperature before loading onto a 5\% polyacrylamide gel. The gels were subjected to electrophoresis and dried, and the labeled DNA was visualized by autoradiography 

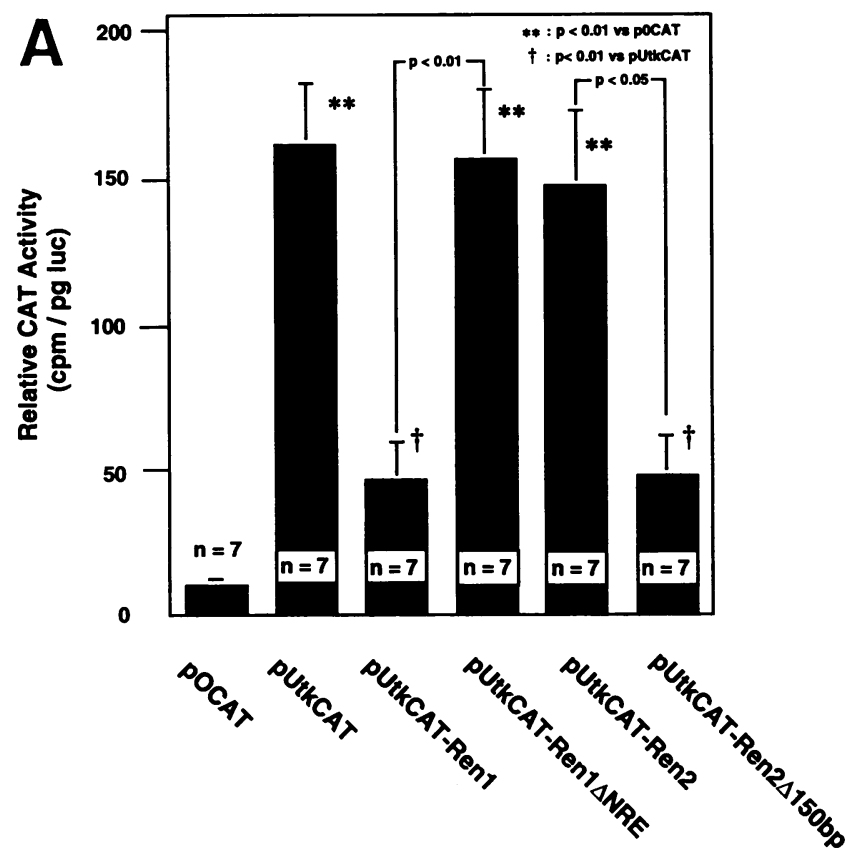

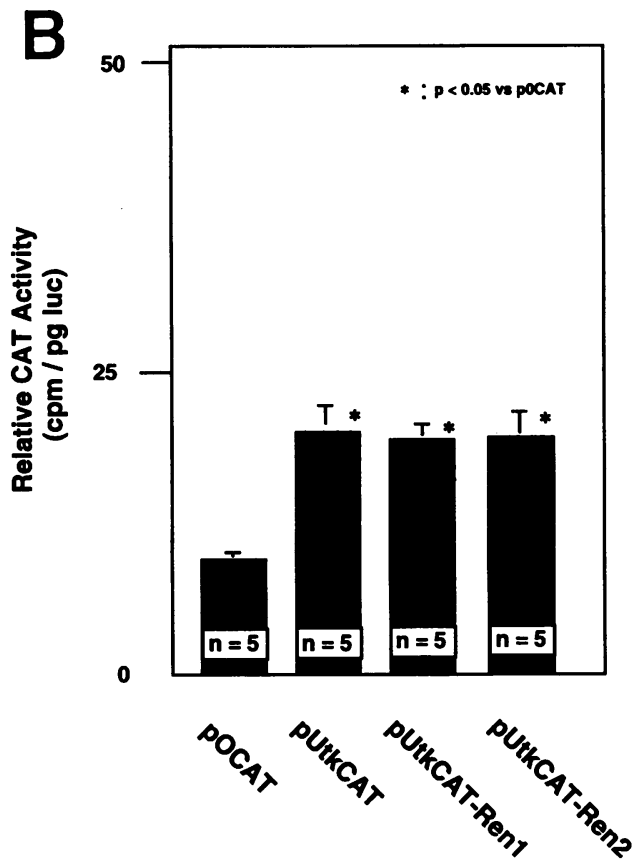

Figure 3. In vivo expression of CAT. The renin-promoter CAT construct was transfected into the SMG $(A)$ or kidney $(B)$ of DBA/2J mice. $3 \mathrm{~d}$ later, the organs were removed, homogenized, and assayed for CAT and luciferase activity. Relative CAT activity (counts per minute/picogram of luciferase) was calculated by dividing the CAT activity in each organ by the corresponding levels of luciferase. ( $A$ ) The transfected vector, pUtkCAT, was readily expressed in the mouse SMG as detected by relative CAT activity compared with that of SMG transfected with p0CAT. The vector, pUtkCAT-Ren1, containing a functional Ren $1^{d}$-NRE abolished CAT expression while the deletion mutant which lacks the NRE (pUtkCAT-Ren1 $\triangle$ NRE) restored CAT expression. The homologous fragment from Ren $2^{d}$ promoter, pUtkCAT-Ren2 had no inhibitory effect on CAT expression while deletion of the 150 -bp insertion from the Ren $2^{d}$ promoter fragment, pUtkCAT-Ren $2 \Delta 150$, abolished CAT expression. ( $B$ ) The vector, pUtkCAT, was significantly expressed in the mouse kidney as detected by CAT activity compared with that of kidney transfected with p0CAT. There was no significant differences in relative CAT activity among pUtkCAT, pUtkCAT-Ren1, and pUtkCAT-Ren2. Results are expressed as mean \pm SE. $n$ for each condition is noted on the graph. Statistical significance was assessed by ANOVA followed by Scheffe's test.

(7). For the competition assay, unlabeled competitor DNA oligonucleotides were preincubated with parallel samples 10 min before the addition of the labeled probe.

\section{Results}

In vivo silencing effect of Ren $1^{d}$-NRE expressed by CAT activity in mouse SMG

SMG transfection. 40 male DBA/2J mice were divided into six groups. Five groups were cotransfected with renin-CAT construct and pGL2-CV $(n=7)$ and one group was transfected with p0CAT and pGL2-CV $(n=5)$. The HVJ-liposomes complex (100 $\mu \mathrm{l})$, containing $10 \mu \mathrm{g}$ of plasmid DNA, was injected directly into the SMG. $3 \mathrm{~d}$ later, these mice were killed and the tissue was removed. The transfected vector, pUtkCAT, was readily expressed in the mouse SMG as detected by CAT activity (3218.2 $\pm 54.8 \mathrm{cpm} / \mathrm{mg}$ protein) compared with that of SMG transfected with p0CAT $(167.9 \pm 12.5 \mathrm{cpm} / \mathrm{mg}$ protein) (ANOVA, mean \pm SE, $P<0.001$ ). The transfection efficiency monitored by luciferase activity showed no statistical difference among the groups $(P=0.68)$. Relative CAT activity was calculated by dividing the CAT activity in each gland by the corresponding levels of luciferase (counts per minute/picogram of luciferase) (Fig. $3 \mathrm{~A}$ ). To test the effect of the Ren $1^{d} \mathrm{XbaI}$ fragment which contains the putative NRE, we examined the expression of pUtkCAT-Ren1. Consistent with our results in cultured JEG-3 cells (6), the presence of the 340-bp XbaI fragment inhibited by fourfold the expression of CAT. We next examined if the NRE sequence was in fact responsible for the inhibition of CAT expression. pUtkCAT-Ren $1 \Delta$ NRE contains the 317-bp XbaI fragment in which the NRE has been deleted. This vector was expressed at levels compatible to pUtkCAT, suggesting a negative effect of the NRE on the activity of the thymidine kinase promoter. The homologous 484-bp XbaI fragment from Ren $2^{d}$ promoter, pUtkCAT-Ren2, had no inhibitory effect on CAT expression compared with pUtkCAT. However, the deletion of the 150-bp insertion adjacent to the NRE from this fragment, pUtkCAT-Ren $2 \Delta 150$, abolished CAT expression $(P<0.01$ vs. pUtkCAT and $P<0.05$ vs. pUtkCAT-Ren2) (Fig. $3 A$ ), demonstrating that the 150 -bp insertion may inhibit the NRE and NREB interaction.

Kidney transfection. 20 male DBA/2J mice were divided into four groups. Mice were cotransfected with each CAT construct (p0CAT, pUtkCAT, pUtkCAT-Ren1, and pUtkCATRen2) coupled with a luciferase reporter construct (pGL2-CV) $(n=5)$. The HVJ-liposomes complex $(300 \mu \mathrm{l})$, containing $30 \mu \mathrm{g}$ of plasmid DNA, was injected into the left kidney via left renal artery. Mice were killed $3 \mathrm{~d}$ later and CAT and luciferase activities in the kidney cortex fragment were measured. The vector, pUtkCAT, was significantly expressed in the mouse kidney as detected by CAT activity $(331.8 \pm 18.8 \mathrm{cpm} / \mathrm{mg}$ protein) compared with that of kidney transfected with p0CAT $(122.9 \pm 13.5 \mathrm{cpm} / \mathrm{mg}$ protein $)(P<0.05)$. The transfection efficiency monitored by luciferase activity showed no statistical difference among the groups $(P=0.77)$ and relative CAT activity was calculated as described previously (counts per mi- 
nute/picogram of luciferase). As shown in Fig. $3 B$, the relative CAT activity was significantly higher in the pUtkCATtransfected kidney as compared with that of p0CAT-transfected kidney, demonstrating successful transfection and expression. However, the CAT activity level of pUtkCAT-transfected kidney was lower than that of pUtkCAT-transfected SMG. This may be due to a lower transfection efficiency of an intraarterial approach and the small population of renin-expressing cells in the kidney. Importantly, there were no significant differences in the relative CAT activities among pUtkCAT-, pUtkCATRen1-, and pUtkCAT-Ren2-transfected kidneys (Fig. $3 B$ ). Therefore, the silencing effect of Ren $1^{d}$-NRE which we observed in the SMG was not observed in the kidney.

In vivo cis- and trans-interactions of renin promoter with double-stranded phosphorothioate Ren ${ }^{d}$-NRE oligonucleotides

To provide further evidence that the interaction of NRE and NREB is involved in tissue-specific gene expression, we employed a decoy approach to modulate the expression of the promoter-CAT chimeric plasmids in vivo $(9,10) .28$ base pair of phosphorothioate oligonucleotides containing the NRE sequence were used because of the reported increase of stability (9). In preliminary experiments using FITC-labeled oligomers, intranuclear fluorescence could be observed $24 \mathrm{~h}$ after injection and was detectable for up to $7 \mathrm{~d}$ (data not shown).

48 male DBA/2J mice were divided into six groups ( $n$ $=8)$. pUtkCAT, pUtkCAT-Ren1, or pUtkCAT-Ren $2 \Delta 150$ was cotransfected with pGL2-CV into the SMG with or without double-stranded phosphorothioate Ren $^{d}{ }^{d}$-NRE oligonucleotides (28-bp, $15 \mu \mathrm{M}$ final concentration) as decoy. $3 \mathrm{~d}$ later, the glands were removed and assayed for CAT and luciferase activity. Consistent with Fig. 3 as well as our previous cell culture studies, pUtkCAT was expressed at levels fourfold higher than pUtkCAT-Ren 1 or pUtkCAT-Ren $2 \Delta 150$. It is important to note that cotransfection of the Ren ${ }^{d}{ }^{d}$-NRE decoy had no effect on the expression of CAT from pUtkCAT (Fig. 4). On the other hand, these oligomers restored the CAT activity of pUtkCATRen 1 to those observed for pUtkCAT $(P<0.05$ vs. pUtkCATRen1 without decoy) (Fig. 4). Moreover, Ren1 ${ }^{d}$-NRE decoy also restored the CAT activity of pUtkCAT-Ren $2 \Delta 150$ to those observed for pUtkCAT $(P<0.05$ vs. pUtkCAT-Ren $2 \Delta 150$ without decoy) $(P<0.05)$ (Fig. 4$)$. These data suggest that exogenously transfected NRE oligonucleotides competed with endogenous NREB, resulting in the inhibition of the binding of NREB to the NRE in the renin gene promoter.

\section{Consensus silencing effect of Ren1 ${ }^{d}$-NRE}

Mouse Ren $1^{d}$-NRE shares homology with the silencer elements in the human renin gene (21), the human c-myc gene (19), the chick lysozyme gene (22), rat collagen II gene (23), and the human $T$ cell receptor gene (24) (consensus sequence [TNN(T/G)TC(C/T)CA(C/G)AGG]). Gel mobility shift assays using ${ }^{32} \mathrm{P}$-labeled $R e n 1^{d}$-NRE probe and nuclear extract from the SMG demonstrated specific complex formation (Fig. $5 A$ ). This binding was competed effectively by $\operatorname{Ren} 1^{d}$-NRE oligonucleotides and only slightly less effectively by human cmyc NRE oligonucleotides, but not at all by mouse MHC-I NRE oligonucleotides (an NRE which does not exhibit homology to the Ren $1^{d}$-NRE [20]). When ${ }^{32}$ P-labeled c-myc NRE probe was incubated with SMG nuclear extracts, a similar complex was formed and, again, the Ren ${ }^{d}$-NRE and c-myc NRE effectively competed this complex formation while the MHC-I NRE

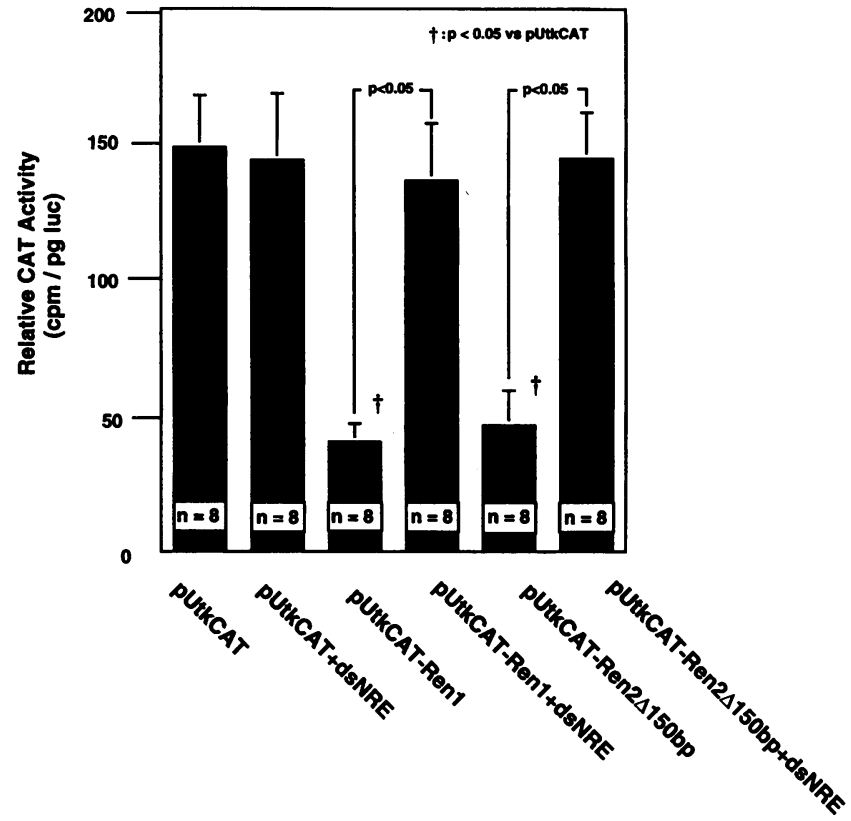

Figure 4. The effect of Ren $1^{d}$-NRE decoy oligonucleotides on CAT expression in vivo. Double-stranded Ren ${ }^{d}$-NRE decoy (28-bp oligonucleotides, $15 \mu \mathrm{M}$ final concentration) was cotransfected into the mouse SMG with pGL2-CV plus either pUtkCAT, pUtkCAT-Ren1, or pUtkCAT-Ren $2 \Delta 150.3 \mathrm{~d}$ later, the glands were removed and assayed for CAT and luciferase activity. Cotransfection of the Ren $1^{d}$-NRE decoy had no effect on the expression of CAT from pUtkCAT. On the other hand, these oligomers restored the CAT activity of pUtkCAT-Ren1 to those observed for pUtkCAT. Moreover, $\operatorname{Ren}^{d}{ }^{d}$-NRE decoy also restored the CAT activity of pUtkCAT-Ren $2 \Delta 150$ to those observed for pUtkCAT. Results are expressed as mean \pm SE. $n$ for each condition is noted on the graph. Statistical significance was assessed by ANOVA followed by Scheffe's test.

was ineffective (Fig. 5 B). The c-myc NRE was slightly less effective at heterologous competition (versus ${ }^{32} \mathrm{P}$-labeled Ren $1^{d}$-NRE) than at the homologous competition (versus ${ }^{32} \mathrm{P}$ labeled c-myc NRE) whereas the Ren $1^{d}$-NRE was equally effective. This suggested that the mouse SMG NREB bound more strongly to the Renl ${ }^{d}$-NRE than to the human c-myc NRE, presumably due to minor sequence differences in the NREs.

We examined whether these double-stranded NRE oligonucleotides would influence the CAT expression regulated by renin gene NRE in vivo. 30 male DBA/ $2 \mathrm{~J}$ mice were divided into five groups $(n=6)$. pUtkCAT-Ren1 plus pGL2-CV was cotransfected with or without phosphorothioate NRE oligonucleotides (28-bp, $15 \mu \mathrm{M}$ final concentration) into the SMG. 3 $\mathrm{d}$ later, the glands were removed and assayed for CAT and luciferase activity. Ren $1^{d}$-NRE decoy restored the expression of pUtkCAT-Ren1 (Fig. 6). Interestingly, human c-myc NRE decoy also restored CAT expression from pUtkCAT-Ren1 ( $P$ $<0.05$ vs. pUtkCAT-Ren1). In contrast, we did not observe any effect when mouse MHC-I NRE oligonucleotides were cotransfected with pUtkCAT-Ren1 (Fig. 6). These results would suggest that Ren $1^{d}$-NRE and human c-myc NRE, but not the negative element from mouse MHC-I, may belong to the same family of silencers that regulate tissue-specific gene expression.

\section{Discussion}

Renin is synthesized in high quantities in the juxtaglomerular cells of the kidney. In addition, there is also evidence for extra- 


\section{A PROBE: [32P]-REN1d NRE}
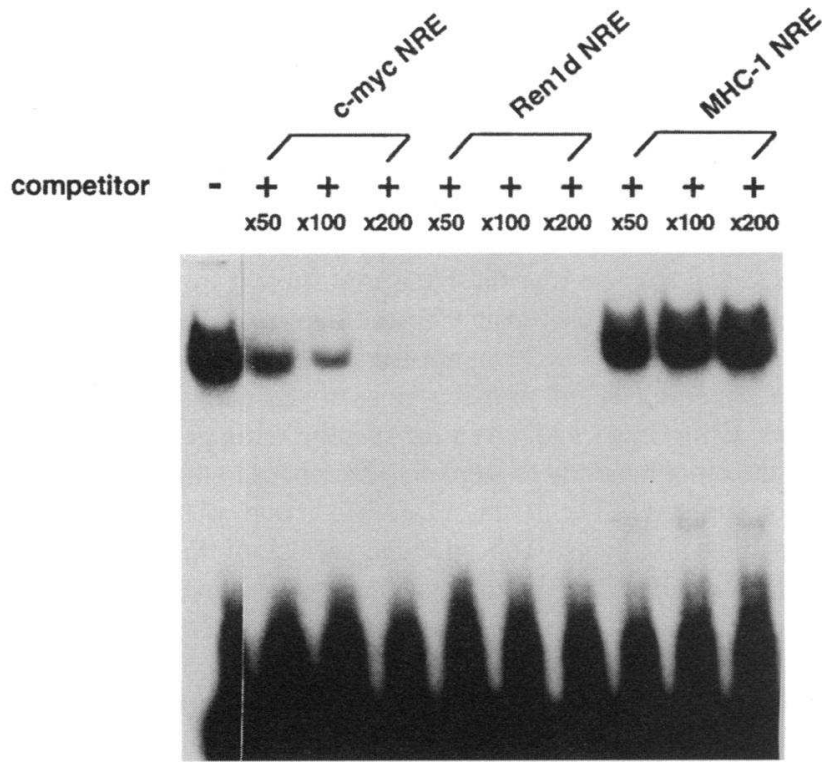

B PROBE: [ ${ }^{32}$ P]-c-myc NRE
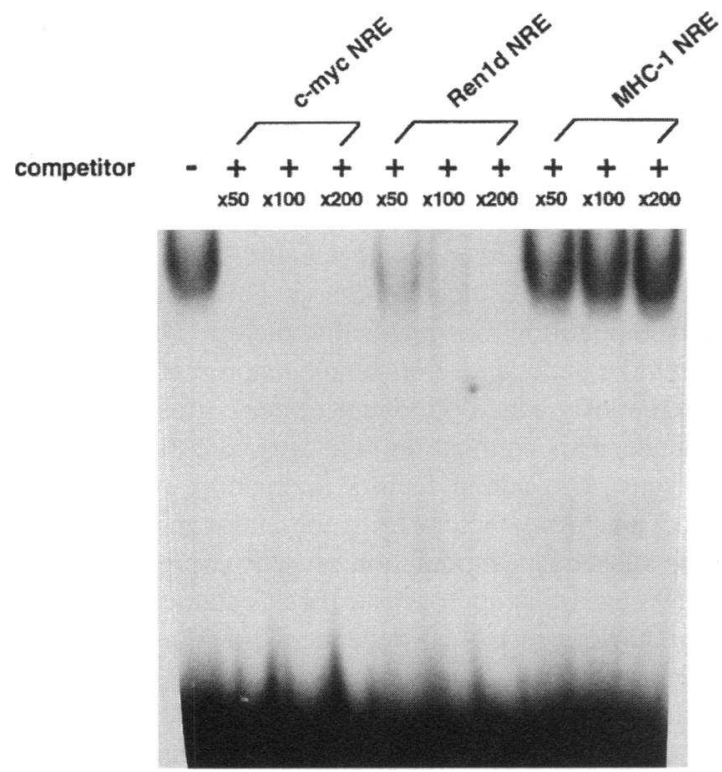

Figure 5. The existence of specific nuclear protein binding with Ren $1^{d}$ NRE. ( $A$ ) Gel mobility shift assays using ${ }^{32}$ P-labeled Ren $1^{d}$-NRE probe and nuclear extract from the SMG (10 $\mu \mathrm{g})$ demonstrated specific complex formation. This binding was competed by Ren $1^{d}$-NRE oligonucleotides and human c-myc NRE oligonucleotides. However, oligonucleotides containing the negative element found in the mouse MHC-I NRE (which does not exhibit homology to the Ren $1^{d}$-NRE) did not compete NRE-NREB complex formation. $(B)$ Gel mobility shift assays using ${ }^{32} \mathrm{P}$-labeled c-myc NRE probe and nuclear extract from the SMG (10 $\mu \mathrm{g})$ also demonstrated specific complex formation, which was competed by Ren $1^{d}$-NRE oligonucleotides but not by the MHC-I NRE oligonucleotides.

renal renin expressions both in experimental animals as well as humans $(3,25)$. This is particularly evident in the mouse in which extrarenal tissues such as SMG, adrenal gland, testis, and ovary express renin in significant concentrations which are un-

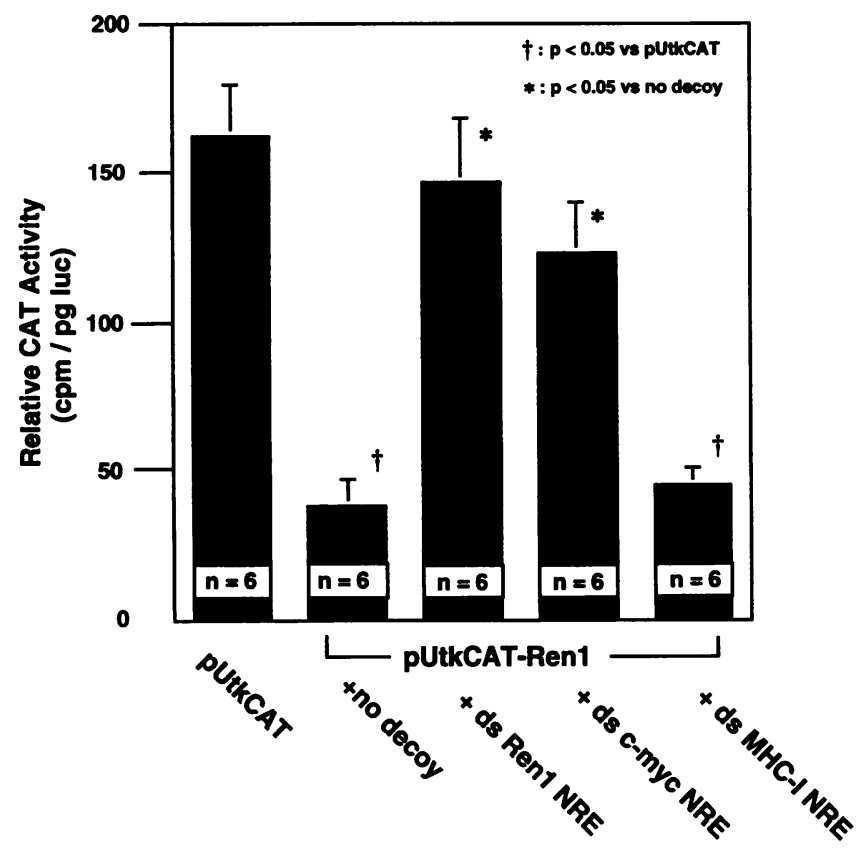

Figure 6. The effect of c-myc NRE and MHC-I NRE decoy oligonucleotides on CAT expression in vivo. pUtkCAT-Ren1 plus pGL2-CV was cotransfected with or without double-stranded phosphorothioate NRE oligonucleotides (28-bp, $15 \mu \mathrm{M}$ final concentration). $3 \mathrm{~d}$ later, the glands were removed and assayed for CAT and luciferase activity. As in Fig. 5, Ren $1^{d}$-NRE decoy restored the expression of pUtkCAT-Ren1. Human c-myc NRE decoy also restored CAT expression from pUtkCAT-Ren1. In contrast, no effect on CAT expression was observed when mouse MHC-I NRE oligonucleotides were cotransfected with pUtkCAT-Ren1. Results are expressed as mean \pm SE. $n$ for each condition is noted on the graph. Statistical significance was assessed by ANOVA followed by Scheffe's test.

der hormonal and genetic control. While some of the extrarenal renin in the mouse is due to the expression of a duplicated gene that is present in certain strains (e.g., DBA/2J), even the single renin gene mouse strains (e.g., C57 BL/10J) express renin in sites distinct from the kidney (26). Although the human genome only contains one renin gene, we and others have shown that several extrarenal tissues in the human (i.e., adrenal, ovary, and placenta) clearly express renin messenger RNA and synthesize the protein $(25,27,28)$. The study of mouse renin gene expression may provide insight into tissue-regulated expression of human renin gene. A detailed comparison of promoter structure and function should be useful in generating a hypothesis of tissue-regulated expression that can be tested further in humans.

The identification of the molecular mechanisms by which the renin gene is regulated has relied on transient transfection experiments in cultured cells. In this approach, various fragments of the renin regulatory region are fused to reporter genes and the constructs introduced into the cells. In theory this approach should yield valuable information but in practice this method suffers from the lack of appropriate renin-expressing cells. For example, Ekker et al. (29) transfected constructs containing the promoter region of the mouse renin genes fused to CAT into five cell lines that do not express renin (baby hamster kidney, L cells, Chinese hamster ovary, JEG-3, and a human mammary tumor cell line). They reported that the promoter regions of these genes were inactive in noncognate cells unless a functional enhancer (SV40) was coupled to the constructs. 
We observed similar results, finding that it was necessary to fuse the renin gene regulatory regions to a thymidine kinase promoter which was in turn linked to a reporter gene to create a functional expression vector. This construct allowed an examination of the putative regulatory regions of the renin gene. Our previous biochemical and in vitro studies suggested that tissueregulated expression is dependent on a complex interaction of positive and negative transcriptional factors competing for a specific cis-region (CRE/NRE) in the $5^{\prime}$ region of the renin gene $(7,8)$. In the kidney the positive factor, CREB (43 kD), binds to the cis-element under basal conditions, blocking the binding of the NREB ( $72 \mathrm{kD}$ ). This allows basal, constitutive expression of the renin gene. In extrarenal sites such as the SMG, the NREB is able to bind effectively to the region, since the CREB is inactivated by an inhibitory protein present in the extrarenal tissue, thereby inhibiting renin expression (7). Some strains of mice (e.g., DBA/2J) contain a second renin gene $\left(\operatorname{Ren} 2^{d}\right)$ which exhibits a different pattern of tissue expression than the wild-type gene $\left(\operatorname{Ren} 1^{d}\right)$. The $\operatorname{Ren} 2^{d}$ but not the Ren $1^{d}$ gene is expressed in the SMG, while both are expressed in the kidney (5). Our data demonstrated that $\operatorname{Ren} 2^{d}$ contains a 150 bp insertion which interferes with the function of the NRE, thus allowing expression in the SMG $(6,8)$. Thus, the regulation of renin expression at this sequence is similar to the dynamic interaction of nuclear factor- $\kappa \mathrm{B}, \mathrm{v}-\mathrm{rel}$, and inhibitor- $\kappa \mathrm{B}$ or I$\kappa \mathrm{B}$ at the region of the $\kappa \mathrm{B}$ sequence (30) first identified in the $\kappa$ immunoglobulin gene.

To circumvent the caveats associated with transient culture experiments, many investigators have used transgenic animal studies. This approach provides the opportunity to examine the expression of transgenes temporally in all tissues. Under appropriate conditions the introduced genes exhibit regulated expression characteristics of the endogenous gene. To date, several groups have undertaken studies using transgenic animals containing various renin genes and constructs. However, some of the conflicting reports have been published concerning the sequences necessary for proper tissue expression $(4,31-33)$.

A technologic advancement in the studies of promoter function has come with the advent of in vivo gene transfer techniques which have recently been used to examine in vivo the sequences necessary for proper gene expression. Previous investigators have reported the feasibility of direct gene transfer into the heart or arterial wall for studying in vivo the sequences necessary for proper gene regulation (34-36). For example, Kitsis et al. (34) showed that a construct containing the $\alpha$ cardiac myosin heavy chain gene promoter driving the expression of CAT was expressed in the rat heart but not in skeletal muscle, demonstrating proper tissue-specific expression. Many methods for the introduction of DNA in vivo have been proposed including viral gene transfer using retrovirus or adenovirus, liposomal gene transfer with cationic liposome (lipofectin), or direct injection of the DNA. We have recently developed a highly efficient and simple method of DNA transfer using the HVJ-liposome method (11). This method is manyfold more efficient than the standard liposome method (14) and does not need complicated plasmid construction as retrovirus or adenovirus transfection requires. Using this gene transfer approach, we have shown that the renin promoter-CAT chimeric plasmids were expressed in a qualitatively similar fashion in vivo to that observed in cell culture. Importantly, the results differed quantitatively in that the magnitude of the inhibition of CAT expression was significantly greater in vivo than that observed in cell culture (6). In vivo, pUtkCAT-Ren1 was expressed at only $10-15 \%$ of the level of pUtkCAT (after subtraction of the background levels as measured by p0CAT), while in cell culture the decrease was on the order of $50-60 \%$. Moreover, the expression of pUtkCAT-Ren1 in vivo was not statistically different from that observed for p0CAT, suggesting that the presence of the NRE in the XbaI fragment completely suppressed CAT expression. Thus, the effects of the NRE on the expression of CAT from the thymidine kinase promoter are similar to the magnitude of (endogenous) suppression of Renl ${ }^{d}$ relative to $R e n 2^{d}$ and suggest that the NRE is sufficient to suppress renin expression. The discrepancy in the data may be explained by the fact that JEG-3 cell is a noncognate cell line which does not express renin (6) and probably does not contain the appropriate trans-acting factors required for specific renin gene expression. On the other hand, there were no differences in the CAT activity among pUtkCAT-, pUtkCAT-Ren1-, and pUtkCAT-Ren2transfected kidneys. The silencing effect of Ren ${ }^{d}$ promoter which we observed in the SMG was not functioning in the kidney. This is compatible with the fact that Ren1 ${ }^{d}$ and $R e n 2^{d}$ genes are expressed equally in the kidney. These results further illustrate the necessity of examining the importance of cis- and trans-interactions in vivo as well as in cell culture. Similar conclusions were drawn by Kitsis et al. (34) and Buttrick et al. (37) in examinations of the cis-elements controlling cardiac myosin expression. One may question the use of the thymidine kinase promoter rather than the endogenous renin promoter. The use of this promoter does necessitate that the conclusions with respect to renin gene expression be carefully stated. On the other hand, it allows potentially a wider interpretation with respect to other genes.

An approach to test the validity of cis- and trans-acting factors interaction involved in tissue-specific gene expression is the transcriptional factor decoy method. This assay is based on the in vivo competition for trans-acting factors. The competition is between the endogenous cis-elements present in a target gene and exogenously added oligonucleotides corresponding to that $c i s$-sequence. This competition will attenuate the authentic cis-elements and trans-acting factors interaction, resulting in the removal of the trans-factors from the endogenous cis-element with the subsequent modulation of gene expression. Bielinska et al. (9) used this approach to block octamer transcription factor or nuclear factor- $\kappa \mathrm{B}$-dependent trans-activation in B cells. Sullenger et al. (10) reported that overexpression of trans-activation response element containing sequences rendered a CD4-positive human T lymphoid cell resistant to human immunodeficiency virus replication. Previously, we have hypothesized, based on the sequence homology, that the mouse renin NRE belonged to a family of negative elements that were found in numerous and disparate genes such as human renin, human $\mathrm{T}$ cell receptor, chicken lysozyme, and rat collagen II (6). In this study, we have furthered the hypothesis by demonstrating that the NRE sequences are functionally similar in that both the renin and c-myc NRE exhibit decoy properties with respect to the SMG expression of pUtkCAT-Ren1, while an unrelated negative element from the MHC-I gene had no effect. Whether the binding proteins which recognize these elements are identical or are also members of a family of proteins is unclear. It is possible that the NREB for mouse renin NRE and the NREB for c-myc NRE are different proteins. However, these proteins have a common DNA binding site, therefore they can recognize consensus NRE sequence. To our knowledge, this is the first validation of the silencing effect of a specific gene sequence demonstrated by direct gene transfer method in vivo. 
Previous reports have used transgenic animals $(38,39)$. Recently, we have purified an NRE binding protein by affinity chromatography using agarose bound NRE (40). The ultimate cloning of the cDNA for this protein should further clarify this point.

In summary, we have identified a DNA sequence in the mouse renin gene (promoter) region which acts in vivo as a silencer. This sequence is conserved in many genes and may function as a consensus silencer in gene regulation. Our data demonstrate that in vivo gene transfer technique using the HVJliposome coupled with transcriptional factor decoy approach may provide a useful method for the general investigation of cis- and trans-elements interaction in tissue-specific gene regulation in vivo.

\section{Acknowledgments}

We thank Dr. Naruya Tomita for technical assistance and Melinda Hing for preparing the manuscript.

This work is supported by National Institutes of Health grants HL46631, HL-35252, HL-35610, HL-48638, and HL-07708, and the American Heart Association Bugher Foundation Center for Molecular Biology in the Cardiovascular System. Takehiko Yamada is the recipient of an American Heart Association California Affiliate Postdoctoral Fellowship Award. Victor J. Dzau is the recipient of National Institutes of Health Merit Award HL-35610.

\section{References}

1. Williams, T. M., D. Moolten, J. Burlein, J. Romano, R. Bhaerman, A. Godillot, M. Mellon, F. J. Rauscher III, and K. A. Kant. 1991. Identification of a zinc finger protein that inhibits IL-2 gene expression. Science (Wash. DC). 254:1791-1794.

2. Leiden, J. M. 1993. Transcriptional regulation of $T$ cell receptor genes. Annu. Rev. Immunol. 11:539-570.

3. Dzau, V. J., D. Burt, and R. E. Pratt. 1988. Molecular biology of the reninangiotensin system. Am. J. Physiol. 255:F563-F573.

4. Sigmund, C. D., and K. W. Gross. 1991. Structure, expression, and regulation of the murine renin genes. Hypertension (Dallas). 18:446-457.

5. Field, L. J., and K. W. Gross. 1985. Ren1 and Ren2 loci are expressed in mouse kidney. Proc. Natl. Acad. Sci. USA. 82:6196-6200.

6. Barrett, G., M. Horiuchi, M. Paul, R. E. Pratt, N. Nakamura, and V. J. Dzau. 1992. Identification of negative regulatory element involved in tissuespecific expression of mouse renin genes. Proc. Natl. Acad. Sci. USA. 89:885889.

7. Horiuchi, M., R. E. Pratt, N. Nakamura, and V. J. Dzau. 1993. Distinct nuclear proteins competing for an overlapping sequence of cAMP and negativeregulatory elements regulate tissue-specific mouse renin gene expression. J. Clin. Invest. 92:1805-1811.

8. Nakamura, N., D. Burt, M. Paul, and V. J. Dzau. 1989. Negative control element and cAMP responsive sequences in the tissue-specific expression of mouse renin genes. Proc. Natl. Acad. Sci. USA. 86:56-59.

9. Bielinska, A., R. A. Shivdasani, L. Q. Zhang, and G. J. Nabel. 1990 Regulation of gene expression with double-stranded phosphorothioate oligonucleotides. Science (Wash. DC). 250:997-1000.

10. Sullenger, B. A., H. F. Gallardo, G. E. Ungers, and E. Giboa. 1990 Overexpression of TAR sequences renders cells resistant to human immunodeficiency virus replication. Cell. 63:601-608.

11. Kaneda, Y., K. Iwai, and Y. Uchida. 1989. Increased expression of DNA coincidenced with nuclear protein in adult rat liver. Science (Wash DC). 243:375-378.

12. Isaka, Y., Y. Fujiwara, N. Ueda, Y. Kaneda, T. Kamada, and R. Imai. 1993. Glomerulosclerosis induced by in vivo transfection of transforming growth factor- $\beta$ or platelet-derived growth factor gene into the rat kidney. J. Clin. Invest. 92:2597-2601.

13. Morishita, R., G. H. Gibbons, K. E. Ellison, M. Horiuchi, M. Nakajima, L. Zhang, Y. Kaneda, T. Ogihara, and V. J. Dzau. 1993. Single intralumina delivery of antisense CDC2 kinase and PCNA oligonucleotides results in chronic inhibition of neointimal hyperplasia. Proc. Natl. Acad. Sci. USA. 90:8474-8478.

14. Morishita, R., G. H. Gibbons, Y. Kaneda, T. Ogihara, and V. J. Dzau. 1993. Novel and effective gene transfer technique for study of vascular renin angiotensin system. J. Clin. Invest. 91:2580-2585.
15. Prost, E., and D. D. Moore. 1986. CAT vectors for analysis of eukaryotic promoters and enhancers. Gene (Amst.). 45:107-111.

16. Seed, B., and J.-Y. Sheen. 1988. A simple phase-extraction assay for chloramphenicol acyltransferase activity. Gene (Amst.). 67:171-177.

17. Bradford, M. 1976. A rapid and sensitive method for the quantitation of microgram quantities of protein utilizing the principle of protein-dye binding. Anal. Biochem. 42:248-254.

18. Brasier, A. R., J. E. Tate, and J. F. Habener. 1989. Optimized use of the firefly luciferase assay as a reporter gene in mammalian cell lines. Biotechniques. 7:1116-1122.

19. Hay, N., M. Takimoto, and J. M. Bishop. 1989. A fos protein is present in a complex that binds a negative regulator of MYC. Genes \& Dev. 3:293-303.

20. Flanagan, J. R., M. Murata, P. A. Burke, Y. Shirayoshi, E. Appella, P. A.

Sharp, and K. Ozato. 1991. Negative regulation of the major histocompatibility complex class I promoter in embryonal carcinoma cells. Proc. Natl. Acad. Sci. USA. 88:3145-3149.

21. Burt, D. W., N. Nakamura, P. Kelley, and V. J. Dzau. 1989. Identification of negative and positive regulatory elements in the human renin gene. J. Biol. Chem. 264:7357-7362.

22. Baniahmad, A., M. Muller, C. H. Steiner, and R. Renkawitz. 1987. Activity of two different silencer elements of the chicken lysozyme gene can be compensated by enhancer elements. EMBO (Eur. Mol. Biol. Organ.) J. 6:2297-2303.

23. Savagner, P., T. Miyashita, and Y. Yamada. 1990. Two silencers regulate the tissue-specific expression of the collagen II gene. J. Biol. Chem. 265:66696674.

24. Winoto, A., and D. Baltimore. 1989. Alpha beta lineage-specific expression of the alpha T cell receptor gene by nearly silencers. Cell. 59:649-655.

25. Baxter, J. D., K. Duncan, W. Chu, M. N. G. James, R. B. Russell, M. A.

Haidar, F. M. DeNoto, W. Hsueh, and T. L. Reudelhuber. 1991. Molecular biology of human renin and its gene. Recent Prog. Horm. Res. 47:211-258.

26. Mullins, J. J., D. W. Burt, J. P. Windass, P. Mataik, H. George, and W. J. Brammer. 1982. Molecular cloning of two distinct renin genes from the DBA/ 2 mouse. EMBO (Eur. Mol. Biol. Organ.) J. 1:1461-1466.

27. Lenz, T., G. D. James, J. H. Laragh, and J. E. Sealey. 1991. Prorenin secretion from human placenta perfused in vitro. Am. J. Physiol. 260:E876-E882.

28. Paul, M., J. Wagner, and V. J. Dzau. 1993. Gene expression of the reninangiotensin system in human tissues. Quantitative analysis by the polymerase chain reaction. J. Clin. Invest. 91:2058-2064.

29. Ekker, M., D. Tronik, and F. Rougeon. 1989. Extra-renal transcription of the renin genes in multiple tissues of mice and rats. Proc. Natl. Acad. Sci. USA. 86:5155-5158.

30. Ballard, D. W., W. H. Walker, S. Doerre, P. Sista, J. A. Molltor, E. P. Dixon, N. J. Peffer, M. Hannink, and W. C. Greene. 1990. The v-rel oncogene encodes a $\kappa \mathrm{B}$ enhancer binding protein that inhibits NF- $\kappa \mathrm{B}$ function. Cell. 63:803814

31. Tronik, D., M. Dreyfus, C. Babinet, and F. Rougeon. 1987. Regulated expression of the Ren-2 gene in transgenic mice derived from parental strains carrying only the Ren-1 gene. EMBO (Eur. Mol. Biol. Organ.) J. 6:983-987.

32. Mullins, J. J., C. D. Sigmund, C. Kane-Haas, C. Wu, F. Pacholec, Q. Zeng, and K. W. Gross. 1988. Studies on the regulation of renin genes using transgenic mice. Clin. Exp. Hypertens. 10:1157-1167.

33. Sola, C., D. Tronik, M. Dreyfus, C. Babinet, and F. Rougeon. 1989. Reninpromoter SV40 large T-antigen transgenes induce tumors irrespective of normal cellular expression of renin genes. Oncogene Research. 5:149-153.

34. Kitsis, R., P. Buttrick, E. NaNally, M. Kaplan, and L. Leinwand. 1991. Hormonal modulation of a gene injected into rat heart in vivo. Proc. Natl. Acad. Sci. USA. 88:4138-4142.

35. Von Harsdorf, R., R. J. Schott, Y. T. Shen, S. F. Vatner, V. Mahdavi, and B. Nadal-Ginard. 1993. Gene injection into canine myocardium as a useful model for studying gene expression in the heart of large mammals. Circ. Res. 72:688695

36. Boyce, F. M., J. C. Stanley, and G. J. Nabel. 1989. Recombinant gene expression in vivo within endothelial cells of the arterial wall. Science (Wash. DC). 244:1342-1344.

37. Buttrick, P. M., A. Kass, R. N. Kitsis, M. Kaplan, and L. A. Leinwand. 1992. Behavior of genes directly injected into the rat heart in vivo. Circ. Res. 70:193-198.

38. Maguire, J. E. W. I. Frels, J. C. Richardson, J. D. Weissman, and D. S. Singer. 1992. In vivo function of regulatory DNA sequence elements of a major histocompatibility complex class I gene. Mol. Cell. Biol. 12:3078-3086.

39. Sawada, S., J. D. Scarborough, N. Killeen, and D. R. Littman. 1994. A lineage-specific transcriptional silencer regulates CD4 gene expression during $\mathrm{T}$ ymphocyte development. Cell. 77:917-929.

40. Horiuchi, M., M. Nakajima, R. Morishita, T. Yamada, M. Mukoyama, R. E. Pratt, and V. J. Dzau. 1994. Purification of the silencer binding protein which is involved in the tissue-specific renin gene expression. J. Hypertens. 12(Suppl. 3):S62a. (Abstr.) 